\title{
Satisfação Dos Estudantes Da Educação Superior Com O Ensino Remoto Durante A Pandemia
}

\section{Higher Education Students' Satisfaction with Remote LearningDuring the Pandemic}

Fernanda Cristina Barbosa Pereira Queiroz Universidade Tecnológica Federal do Paraná E-mail: fernandacbpereira@gmail.com

\section{Christian Luis Da Silva}

Universidade Tecnológica Federal do Paraná

E-mail: christianlsilva76@gmail.com

\section{Nilton Cézar Lima}

Universidade Federal de Uberlândia

E-mail: cesarlim@yahoo.com

\section{Jamerson Viegas Queiroz}

Universidade Federal do Rio Grande do Norte

E-mail: viegasqueiroz@gmail.com

\section{Eduardo Lopes Marques}

Universidade do Centro-Oeste do Paraná

E-mail: eduardomarques@unicentro.br

\section{Carmem Kistemacher Barche}

Universidade Federal do Paraná

E-mail: carmemkistbarche@gmail.com

\section{Diego Derick Oliveira De Medeiros}

Universidade Federal do Rio Grande do Norte

E-mail: diego.derick@hotmail.com

\section{Wecsley Soares De Souza Costa}

Universidade Federal do Rio Grande do Norte

E-mail:wecsley@favoritosupermercados.com.br

\section{RESUMO}

Devido à pandemia da Covid-19 surgiu a necessidade de distanciamento social a fim de evitar o contágio e, como consequência, o ensino remoto (ER) foi adotado como um regime excepcional por quase todas as Instituições de EducaçãoSuperior (IES), no mundo, em 2020. O principal objetivo desta pesquisa é identificar os fatores mais importantes, na percepção dos estudantes, que 
afetaram a satisfação deles com o ER. Este estudo utilizou uma abordagem quantitativa, por um método de amostragem não probabilístico, por meio da análise de 311 questionários respondidos online. O software $R$ foi utilizado para aanálise descritiva e o SmartPLS para analisar os dados recorrendo-se à modelagem de equações estruturais no intuito de examinar a relação entrevariáveis independentes e dependentes. Verificou-se uma relação positiva e significativa entre os constructos, e as duas hipóteses sugeridas foram confirmadas, sendo que $\mathrm{H}_{1}$ : há uma relação positiva entre a percepção dequalidade e a satisfação dos estudantes com o ER e $\mathrm{H}_{2}$ : há uma relação positivaentre as características individuais dos estudantes e a satisfação com o ER. Os resultados da pesquisa contribuem para a discussão acerca do impacto da pandemia na educação superior e agrega conhecimento em relação aos determinantes da satisfação do estudante com o ER.

Palavras chave: Ensino remoto, Satisfação, Estudantes, Pandemia

\section{ABSTRACT}

Due to the Covid-19 pandemic the need for social distancing arose in order to avoid contagion and as a consequence, remote learning (RE) was adopted as an exceptional regime by almost all Higher Education Institutions (HEls), worldwide, in 2020. The main objective of this research is to identify the most important factors, in students' perception, that affected their satisfaction with ER. This studyused a quantitative approach, by a non-probability sampling method, through the analysis of 311 questionnaires answered online. $R$ software was used for descriptive analysis and SmartPLS to analyze the data using structural equation modeling to examine the relationship between independent and dependent variables. There was a positive and significant relationship between the constructs, and the two suggested hypotheses were confirmed, being $\mathrm{H} 1$ : there is a positive relationship between perceived quality and students' satisfaction with RE and $\mathrm{H} 2$ : there is a positive relationship between students' individual characteristics and satisfaction with RE. The research findings contribute to the discussion about the impact of pandemic on higher education and adds knowledge regarding the determinants of student satisfaction with RE.

\section{Keywords: Remote Education, Satisfaction, Students, Pandemic}

\section{INTRODUÇÃO}

O ensino remoto (ER) tornou-se a principal modalidade de ensino e aprendizagem com a declaração, pela Organização Mundial de Saúde (OMS) emmarço de 2020, de situação de pandemia da Covid-19, doença causada pelo novocoronavírus (SarsCov-2). A partir desta realidade, rapidamente, sem planejamento, houve a necessidade de adaptação das aulas presenciais para umnovo modelo, desafiador para a maioria dos docentes e estudantes no mundo.

A necessidade de isolamento social, como estratégia para evitar a propagação do coronavírus, e a preocupação com a saúde da comunidadeacadêmica, fez com que, em pouco tempo, as aulas e avaliações passassem a ocorrer de forma virtual mediadas pelas Tecnologias Digitais da Informação e Comunicação (TDIC).

Verificou-se, a partir deste cenário, a implantação de soluções para que o ensino, anteriormente planejado para o formato presencial, fosse ministrado 
remotamente. Trata-se de uma mudança temporária da entrega de instruções para um modo de entrega alternativo devido à crise e não a busca pela recriação de um ecossistema educacional novo e totalmente robusto (HODGES et al., 2020). Arruda (2020) corrobora com esta visão enfatizando que o ER se diferencia da Educação à Distância (EaD) pelo caráter emergencial, se apropriando das TDIC circunstâncias especificas de atendimento onde outrora existia regularmente a educação presencial. Trata-se, na opinião do autor, de uma estratégia importante para manter o vínculo entre estudantes, professores e demais profissionais da educação, mesmo com limitações.

Neste sentido, o presente trabalho, a partir da construção e aplicação de um questionário online direcionado para estudantes matriculados na educação superior no Brasil, procurou responder à pergunta: Em função da necessidade de distanciamento social, e a implantação de um regime excepcional de oferta de disciplinas, quais as variáveis foram determinantes para a satisfação dos estudantes com o ER? Para responder à questão de pesquisa proposta, o objetivo deste trabalho é identificar os fatores mais importantes, na percepção dos estudantes, que afetaram a satisfação deles com o ER e são apresentados os seguintes objetivos específicos: i) compreender o grau de concordância dos estudantes com aspectos que avaliam o ER; ii) identificar como a percepção de qualidade com o ER afeta a satisfação dos estudantes; iii) identificar como as características individuais afetam a satisfação dos estudantes; iv) sintetizar em um modelo estrutural o relacionamento entre as variáveis.

Como justificativa teórica para o presente estudo, é importante destacar que o ER foi uma solução que as Instituições de Educação Superior (IES) encontraram para não interromper definitivamente as atividades acadêmicas e o tema é, portanto, recente e desafiador. A pesquisa está organizada, além desta introdução, em outras quatro seções: a revisão de literatura foi construída com o intuito de dar suporte teórico à proposição das hipóteses do modelo para avaliar a satisfação dos estudantes universitários com o ER. A metodologia discute questões referentes ao instrumento de coleta, amostra, procedimentos de análise e operacionalização do modelo. A quarta seção é dedicada a análise dos resultados, em que se apresentam o perfil dos respondentes, análise gráfica dasassertivas, os modelos de mensuração e estrutural, onde avalia-se a qualidade doajustamento dos mesmos, assimilando-as às referências bibliográficas que deram suporte ao trabalho. Finalmente, a quinta seção apresenta as conclusões, permitindo reunir as percepções colhidas dos resultados.

\section{REVISÃO DA LITERATURA E DESENVOLVIMENTO DAS HIPÓTESE \\ 2.1 a Pandemia Da Covid-19 E O Ensino Remoto}

As mudanças impostas às instituições educacionais com a Coronavirus Disease (Covid-19) foram muito desafiadoras. Um surto viral encontrado inicialmente em dezembro de 2019 em Wuhan, China, se espalhou rapidamente pelo mundo e logo tornou-se uma pandemia, conforme os critérios da Organização Mundial da Saúde (LIMA et al., 2020).

Neste cenário pandêmico, fez-se necessária a adoção de medidassanitárias emergenciais, tais como o fechamento de fronteiras e estabelecimentoscomerciais

e educacionais, restrições de viagem, quarentena e medidas dedistanciamento social a fim de evitar a propagação do vírus (NICOLA et al., 2020).

As IES, na maior parte dos países, suspenderam as atividades presenciaise adotaram estratégias de ensino e aprendizagem virtuais visando prosseguir com 
as atividades acadêmicas e proteger a saúde dos estudantes, docentes e técnicos administrativos (ARRUDA, 2020; GELLES et al., 2020; GARCÍA-ALBERTI et al., 2021).

As pessoas tiveram pouco tempo para se ajustar e modificar o processo deensino e aprendizado para o sistema remoto. Ao mesmo tempo que os docentesadaptaram os currículos e planos de ensino para uma nova modalidade de aprendizagem, ficou evidenciado em muitos casos as disparidades socioeconômicas e habilidades e competências diferentes entre os estudantes para acessarem os recursos tecnológicos necessários à aprendizagem online (GELLES et al., 2020, RAHIEM, 2020).

No Brasil, a EaD é uma modalidade de ensino que vem se tornando cada vez mais popular e que para ser implantada necessita de uma série de demandas relacionadas à gestão, ao planejamento e à organização. Por sua vez, o ER de emergência pode ser definido como sendo um modelo conceitual de aprendizagem que compreende uma rápida transformação dos cursos presenciaispara o ambiente online, fornecendo soluções improvisadas para acomodar circunstâncias inesperadas (HODGES et al., 2020).

Diante desta conjuntura, visando garantir uma educação de qualidade, muitos docentes deram atenção às atividades que privilegiassem a autonomia dos estudantes, sendo necessário o envolvimento dos alunos para manter um processo de aprendizagem ativo. Por se tratar de uma realidade nova, poucos estudos empíricos conseguiram retratar como ocorreu este engajamento, além dos impactos deste processo de ensino na aprendizagem dos estudantes. Raras também são as pesquisas que relacionaram a qualidade do ER e as características individuais dos estudantes com a satisfação dos mesmos com estamodalidade de ensino e aprendizagem (PURIWAT; TRIPOPSAKUL, 2021).

\subsection{A Qualidade Percebida Pelos Estudantes Durante O Ensino Remoto}

Se, no caso da EaD, a dimensão de qualidade é um conceito complexo e envolve variáveis como apoio institucional, estrutura dos cursos, corpo docente, planejamento, qualidade do sistema e do material, suporte ao estudante, metodologia de ensino e aprendizagem, infraestrutura robusta e confiável são utilizadas para mensurar a qualidade percebida dos cursos (JUNG, 2011; ESFIJANI, 2018); no caso do ER esta complexidade e dificuldade é ainda maior, não havendo consenso em relação às dimensões que influenciam a percepção da qualidade.

Nesta perspectiva, a percepção da qualidade com o ER foi entendida comosendo influenciada pelo caminho metodológico percorrido pelo docente, assim como pelas habilidades e competências dos mesmos em lidar com a situação inesperada de migrar para o ER, na maior parte dos casos, sem capacitação e utilizando recursos próprios com a finalidade de garantir a continuidade doprocesso educacional (ABBASI et al., 2020; ADAMS BECKER et al., 2017).

A continuidade dos estudos de forma remota possibilitou que os cursos de graduação não fossem interrompidos por um longo período. A aprendizagem percebida é potencializada com o desenvolvimento de projetos práticos (Souza et al., 2021). Por se tratar de uma modalidade de ensino mediada pela tecnologia da informação via internet, a dimensão tecnológica também contribuiu para a percepção da qualidade. As IES precisam se concentrar em estudos que 
consideram esta dimensão para aprimorar os cursos (Elumalai et al; 2020;Aldhahi, 2021).

Cabe destacar que, sob o contexto pandêmico, a implantação do ER, se fazia concomitante à intensa propagação do vírus, e, por conseguinte, outras intempéries associadas a aspectos sociais e econômicos perfaziam o composto complexo da migração emergencial no formato de ensino presencial para o remoto. Ou seja, em meio à um vírus de capacidade letal em circulação, que em dada circunstância de convivência restrita como forma de mitigar sua propagação,não só alterou o modo das pessoas se socializarem, como também elevou o temorpela possibilidade de algum ente da família ser contagiado e agravar o convívio familiar, e por conseguinte, colocar a efetividade educacional em plano secundárioou mesmo ter que descartá-lo, seja por parte do estudante quanto pelo docente.

Contudo, o ER foi a solução encontrada e implementada nas IES, e sob o aspecto da percepção da qualidade, coube então constatar se de fato, para o estudante 0 aprendizado foi útil e significativo.

\subsection{As características individuais dos estudantes}

Os cursos online na educação superior utilizam uma abordagem centrada no estudante, enfatizando uma aprendizagem dinâmica e o envolvimento do aluno, além de suas atitudes e habilidades em relação ao domínio da tecnologia (SUN et al., 2008; DEBATTISTA, 2018; ASHWINE; MCVITTY, 2015); além disso,

a interação dos estudantes com seus pares no ambiente de aprendizagem melhora a qualidade do aprendizado (GOH et al., 2017; YEKEFALLAH et al., 2021).

A relação entre a persistência da aprendizagem e a interação do aluno em ambientes de aprendizagem online revelou um efeito moderador (YU et al., 2020). Elumalai et al. (2020) reforça que as características dos estudantes afetam a satisfação com as aulas online. Assim, adotou-se a satisfação como característicaa perscrutar nesta pesquisa. Esta, associada ao objeto deste estudo, quanto à percepção dos estudantes com ER.

\section{SATISFAÇÃO}

Alguns, em alguns estudos anteriores à pandemia, evidenciou-se uma associação positiva entre a satisfação dos estudantes e a aprendizagem obtida pela EaD (ATCHLEY et al., 2013; MAKI et al., 2000). No geral, o que se observou,foi que os alunos consideravam os cursos híbridos ou parcialmente online como mais satisfatórios que aqueles totalmente a distância (COLE et al., 2014).

Outros estudos também demonstraram que, a satisfação do estudante coma EaD está relacionada com o seu nível de habilidades e competências digital, como o envolvimento e interação, apoio e orientação do instrutor e o design do curso. Em geral, fatores pessoais, como personalidade do aluno, atitudes em relação à tecnologia, experiência de uso anterior e habilidade foram observados para influenciar a satisfação dos alunos (BOLLIGER, 2004; ALDHAHI et al., 2021). A autonomia e a percepção dos estudantes a respeito da sua capacidade de realizar o controle das tarefas e conduzir sua aprendizagem são outros fatores essenciais que também afetam a satisfação do aluno SEIVER; TROJA, 2014; ALDHAHI et al., 2021).

Contudo, como apresentado anteriormente, o ER é distinto da EaD e essas diferenças, associada com a necessidade do distanciamento social durante a 
pandemia, justificam a realização de pesquisas com o intuito de investigar aspectos como habilidade, competências e características dos estudantes e as relações da qualidade na satisfação com o ER (SAXENA; BABER; KUMAR, 2021; PURIWAT; TRIPOPSAKUL, 2021). Especificamente no contexto do ensino superior, acredita-se que a satisfação dos estudantes tem uma relação com a percepção da qualidade do curso e também da sua motivação, acessibilidade, dedicação, habilidade e competências em utilizar os recursos tecnológicos. Diante do exposto, tem-se as seguintes hipóteses:

- $\quad H_{1}$ : Há uma relação positiva entre a percepção de qualidade e a satisfação dos estudantes com o ER.

- $\quad H_{2}$ : Há uma relação positiva entre as características individuais dos estudantes e a sua satisfação com o ER.

\section{METODOLOGIA}

Este trabalho utilizou uma abordagem quantitativa de natureza exploratóriodescritiva, visando coletar dados a partir da resposta de indivíduos, para posterior tratamento estatístico. A população da pesquisa é representada por estudantes matriculados na educação superior no Brasil. A amostragem foi não probabilísticae por conveniência e a obtenção da amostra do estudo foi realizada mediante o envio do link do questionário, armazenado na plataforma Survey King, por e-maile redes sociais.

O questionário aplicado foi composto por dois blocos, sendo o primeiro deles formado por 24 assertivas que abordaram as variáveis de análise, e o segundo por questões referentes ao perfil do respondente. As respostas dos estudantes foram realizadas considerando o cenário da pandemia e assim, foi apresentado um parágrafo introdutório destacando que "Diante da situação atualde pandemia e a necessidade de continuação das atividades acadêmicas de forma segura, qual a sua percepção a respeito dos itens avaliados:". Considerando o número mínimo de observações entre cinco e dez respondentespor cada variável (HAIR et al., 2017), para o questionário aplicado, foi obtida umaamostra de 335 respondentes sendo 311 considerados válidos.

As questões sobre a avaliação da satisfação com o ER durante a pandemia utilizaram uma escala Likert de 5 pontos - "Discordo totalmente", "Discordo", "Nem concordo nem discordo", "Concordo", "Concordo totalmente" - que consistiam em assertivas a respeito das variáveis informadas no Quadro 1.

\section{Quadro 1 - Variáveis e indicadores utilizados na pesquisa}

\begin{tabular}{|c|c|c|c|}
\hline $\begin{array}{l}\text { Variáveis } \\
\text { Latentes de } 2^{\text {a }} \\
\text { Ordem }\end{array}$ & $\begin{array}{l}\text { Variáveis } \\
\text { Latentes de } 1^{\text {a }} \\
\text { Ordem } \\
\end{array}$ & $\begin{array}{l}\text { Indicadore } \\
\text { s }\end{array}$ & Descrição \\
\hline \multirow{4}{*}{$\begin{array}{l}\text { Qualidade } \\
\text { percebida }\end{array}$} & \multirow{4}{*}{ Docentes } & DOC1 & $\begin{array}{l}\text { Os professores, de uma maneira geral, } \\
\text { utilizarammetodologias variadas de avaliação }\end{array}$ \\
\hline & & DOC2 & $\begin{array}{l}\text { Os professores se esforçaram para possibilitar } \\
\text { aminha aprendizagem }\end{array}$ \\
\hline & & DOC3 & $\begin{array}{l}\text { Os professores, de uma maneira geral, têm } \\
\text { habilidades para compartilhar o } \\
\text { conhecimentoutilizando tecnologias }\end{array}$ \\
\hline & & DOC4 & $\begin{array}{l}\text { Os professores, de uma maneira geral, utilizaram } \\
\text { diferentes estratégias no processo de ensino } \\
\text { eaprendizagem }\end{array}$ \\
\hline
\end{tabular}




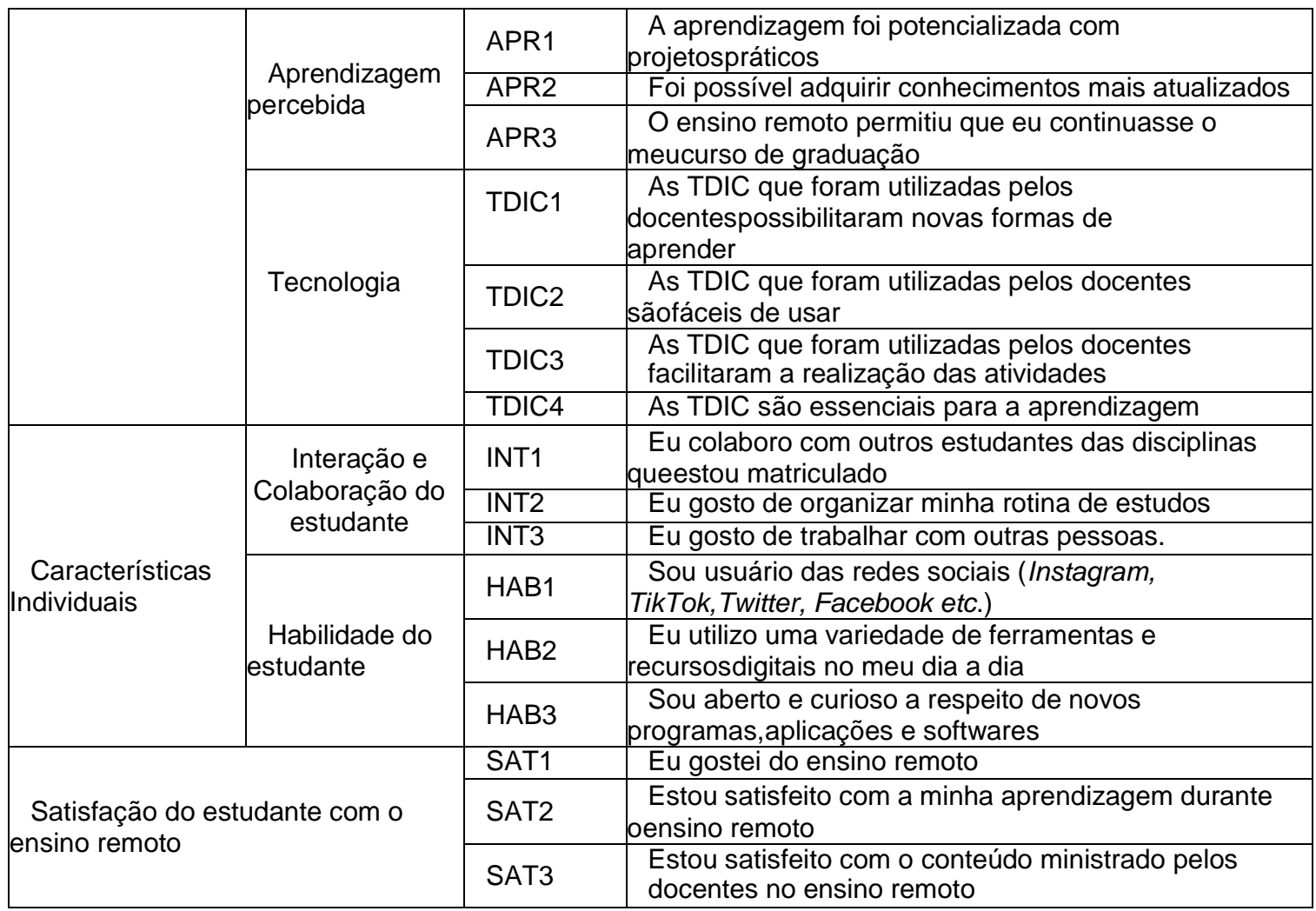

Fonte: Dados da pesquisa, 2021.

Inicialmente realizou-se a análise descritiva do perfil dos estudantes e a seguir, com o software $R$ e o ambiente RStudio foram construídos gráficos considerando os valores relativos das questões preenchidas com a escala Likert. Os eixos à esquerda informaram a soma relativa dos valores "péssimo e ruim" e os eixos à direita indicaram a soma dos valores "Bom" e "Excelente". Os valores regulares são apresentados no centro do gráfico. Foi considerada uma avaliação satisfatória aqueles itens cuja soma de "Bom" e "Excelente" são superiores a 50\%e o restante, ou seja caso a soma de "Péssimo", "Ruim" e "Regular" sejam até $50 \%$, foi considerado como sendo insatisfatório.

Para testar as hipóteses de causalidade entre as variáveis, os dados foram submetidos a um modelo de equações estruturais baseado em ajuste de Mínimos Quadrados Parciais, utilizando o software SmartPLS, versão 3.2.8. As variáveis latentes de primeira ordem foram mensuradas pelos indicadores informados no Quadro 1 e as variáveis latentes de segunda ordem foram criadas reutilizando os indicadores das variáveis originais, uma vez que há pouca diferença em relação a quantidade de indicadores em cada uma (BIDO; SILVA, 2019). O PLS-SEM baseiase no procedimento não-paramétrico de boostrapping (EFRON; E TIBSHIRANI, 1986; DAVISON; HINKLEY, 1997) para testar a significância de vários resultados, tais como: coeficientes estimados para os caminhos, alfa de Cronbach e variância explicada $\left(\mathrm{R}^{2}\right)$. Utilizou-se uma reamostragem de 1000 subamostras.

$\mathrm{Na}$ sequência, a análise do ajuste do modelo é apresentada em duas etapas: avaliação dos modelos de mensuração - para testar a confiabilidade e validade das variáveis latentes utilizadas no modelo - e avaliação do modelo estrutural - que fornece métricas sobre a qualidade de ajuste do modelo, indicando seu grau de capacidade preditiva. 


\section{RESULTADOS}

\subsection{PERFIL DOS RESPONDENTES}

A partir do questionário aplicado, construiu-se o perfil dos respondentes. Amaior parte dos estudantes está matriculada em IES Privadas (201 estudantes correspondendo a $65 \%$ da amostra). Observou-se que, quanto à identidade de gênero, $52 \%$ deles são mulheres; $45 \%$ homens; $1,0 \%$ homem trans; $0,3 \%$ não binário e 1,0\% preferiu não informar.

Em relação à idade, a maioria dos respondentes tem menos de 24 anos, com idade média de 23,25 anos, sendo a faixa etária predominante os estudantesentre 20 a 24 anos de idade (54\%). Foi realizado um teste $t$ de Student para amostras independentes com o objetivo de investigar em que medida as idades eram diferentes entre os respondentes das IES públicas e privadas. Utilizou-se acorreção de Levene, em função das variâncias diferentes. Os resultados demonstraram que a idade dos estudantes das IES privadas é estatisticamente maior (Média $=24,34$; Desvio padrão $=4,64$ ) do que os das IES públicas (Média

$=21,3$; Desvio padrão 2,25) e $(\mathrm{t}(305)=-7,72, \mathrm{p}<0,000)$. O tamanho de efeito da diferença foi médio ( $d$ de Cohen $=0,76$ ).

Quanto à organização acadêmica, 38\% dos respondentes estão matriculados em Universidades e $26 \%$ em Faculdades. Houve respondentes de todas as regiões do país, sendo a maioria da região sudeste (52\%).

Quadro 2 - Perfil dos respondentes

\begin{tabular}{|c|c|c|c|c|c|}
\hline \multicolumn{2}{|c|}{ Variáveis / Categoria administrativa } & Pública & $\begin{array}{r}\text { Privad } \\
a\end{array}$ & $\begin{array}{r}\text { Total } \\
(\mathrm{N})\end{array}$ & \begin{tabular}{r|} 
Total \\
$(\%)$
\end{tabular} \\
\hline \multirow{6}{*}{ Identidade de gênero } & Mulher & 55 & 108 & 163 & $52 \%$ \\
\hline & Homem & 51 & 90 & 141 & $45 \%$ \\
\hline & Homem trans & 2 & 1 & 3 & $1 \%$ \\
\hline & Não binário & & 1 & 1 & $0 \%$ \\
\hline & Não informou & 2 & 1 & 3 & $1 \%$ \\
\hline & Total & 110 & 201 & 311 & $100 \%$ \\
\hline \multirow{5}{*}{ Idade } & Até 19 anos & 27 & 24 & 51 & $16 \%$ \\
\hline & de 20 a 24 anos & 78 & 89 & 167 & $54 \%$ \\
\hline & de 25 a 29 anos & 5 & 73 & 78 & $25 \%$ \\
\hline & Mais de 29 anos & 0 & 15 & 15 & $5 \%$ \\
\hline & Média & 21,3 & 24,34 & 23,25 & \\
\hline \multirow{5}{*}{$\begin{array}{l}\text { Organização } \\
\text { Acadêmica }\end{array}$} & Universidade & 76 & 42 & 118 & $38 \%$ \\
\hline & Faculdade & & 82 & 82 & $26 \%$ \\
\hline & Centro Universitário & & 77 & 77 & $25 \%$ \\
\hline & IF Cefet & 34 & & 34 & $11 \%$ \\
\hline & Total & 110 & 201 & 311 & $100 \%$ \\
\hline \multirow{6}{*}{ Região } & Sudeste & 51 & 111 & 162 & $52 \%$ \\
\hline & Sul & 16 & 30 & 46 & $15 \%$ \\
\hline & Centro Oeste & 9 & 18 & 27 & $9 \%$ \\
\hline & Nordeste & 26 & 30 & 56 & $18 \%$ \\
\hline & Norte & 8 & 12 & 20 & $6 \%$ \\
\hline & Total & 110 & 201 & 311 & $100 \%$ \\
\hline
\end{tabular}

Fonte: Dados da pesquisa, 2021.

\subsection{ANÁLISE DAS ASSERTIVAS}

Para a variável latente "Qualidade percebida" predominou a concordância 
com as assertivas informadas na Figura 01, com destaque para a percepção que os estudantes tiveram em relação aos esforços dos docentes para possibilitar o ensino e a aprendizagem (92\%), no papel que o ER teve ao possibilitar que os cursos de graduação fossem continuados (75\%) e no uso pelos docentes de diferentes estratégias visando o compartilhamento do conhecimento diante do cenário de distanciamento social (73\%). As maiores discordâncias foram encontradas no uso de metodologias de avaliação variadas $(29 \%)$ e no papel essencial das TDIC para a aprendizagem (27\%).

Figura 01 - Qualidade percebida - Concordância e discordância em \%

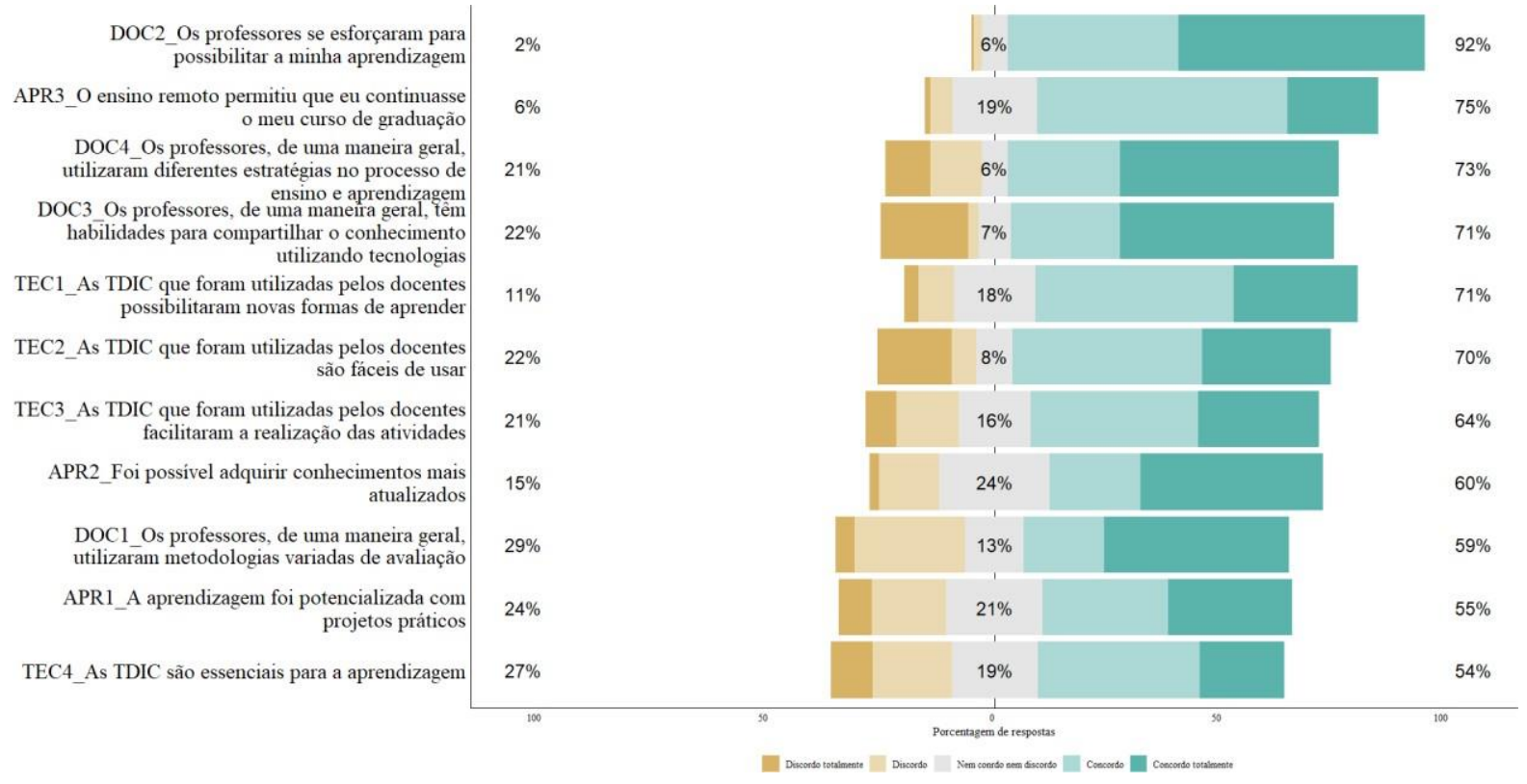

Fonte: Dados da pesquisa, 2021.

Em relação às "Características individuais" dos estudantes, verificou-se a concordância com todas as assertivas informadas. Conforme ilustra a Figura 02, a maior parte dos estudantes concordou com as afirmações a respeito de serem usuários das redes sociais, e ainda por utilizarem ferramentas e recursos digitaise, serem curiosos e colaborativos. Além de gostarem de trabalhar em equipe e organizar a rotina de estudos. Registra-se que $70 \%$ dos respondentes tem até 24 anos de idade. 
Figura 02 - Características individuais - Concordância e discordância em \%

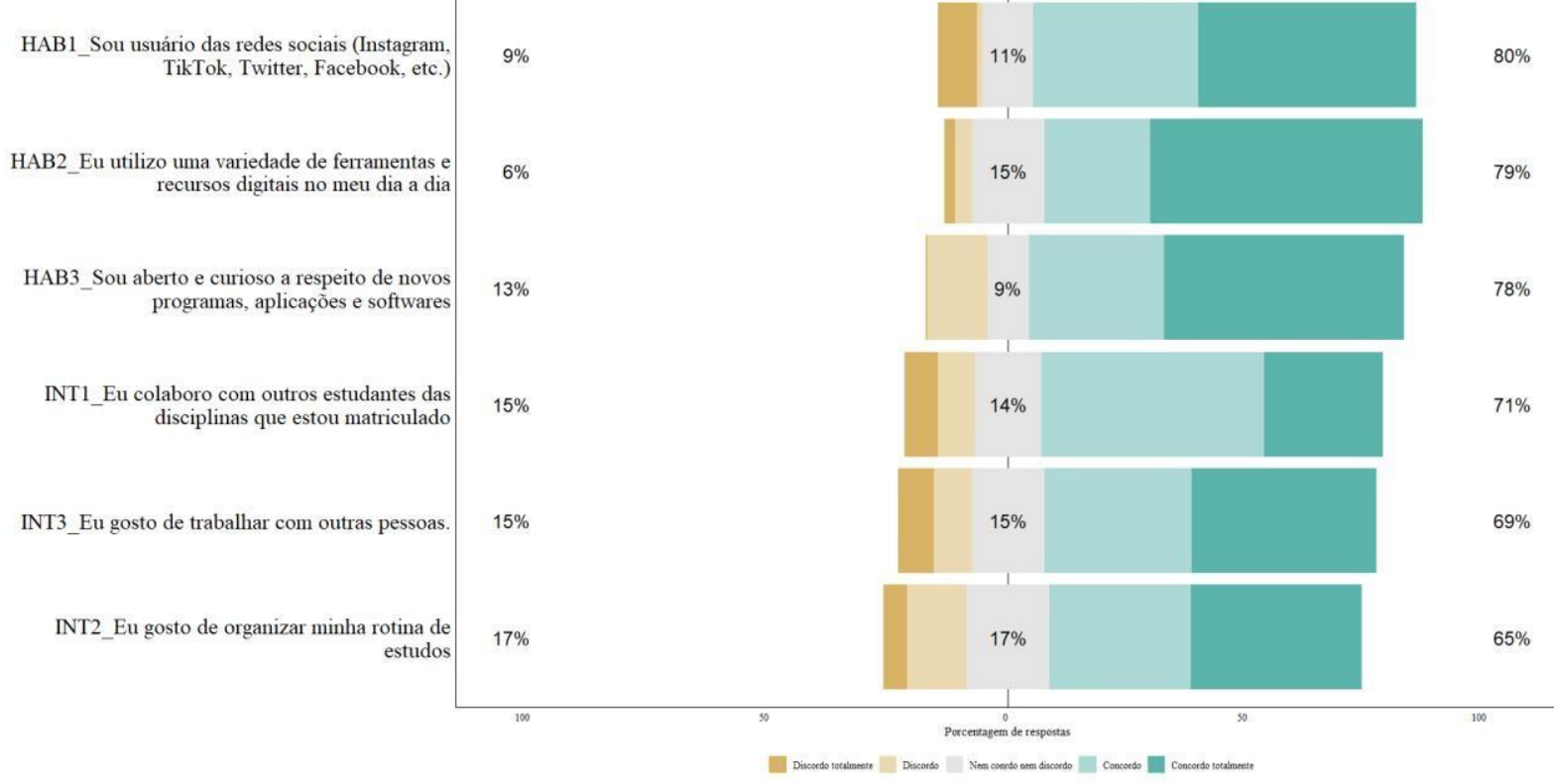

Fonte: Dados da pesquisa, 2021.

Por fim, em relação à "Satisfação" dos estudantes, houve também concordância com todas as assertivas informadas. Verifica-se na Figura 03 que diante do cenário de pandemia, os estudantes estão satisfeitos com o conteúdo ministrado pelos docentes no ER (77\%), gostaram do ER (72\%) e estão satisfeitos com a aprendizagem durante o ER, sendo este indicador o que apresentou o menor percentual de concordância (58\%)

Figura 03 - Satisfação - Concordância e discordância em \%

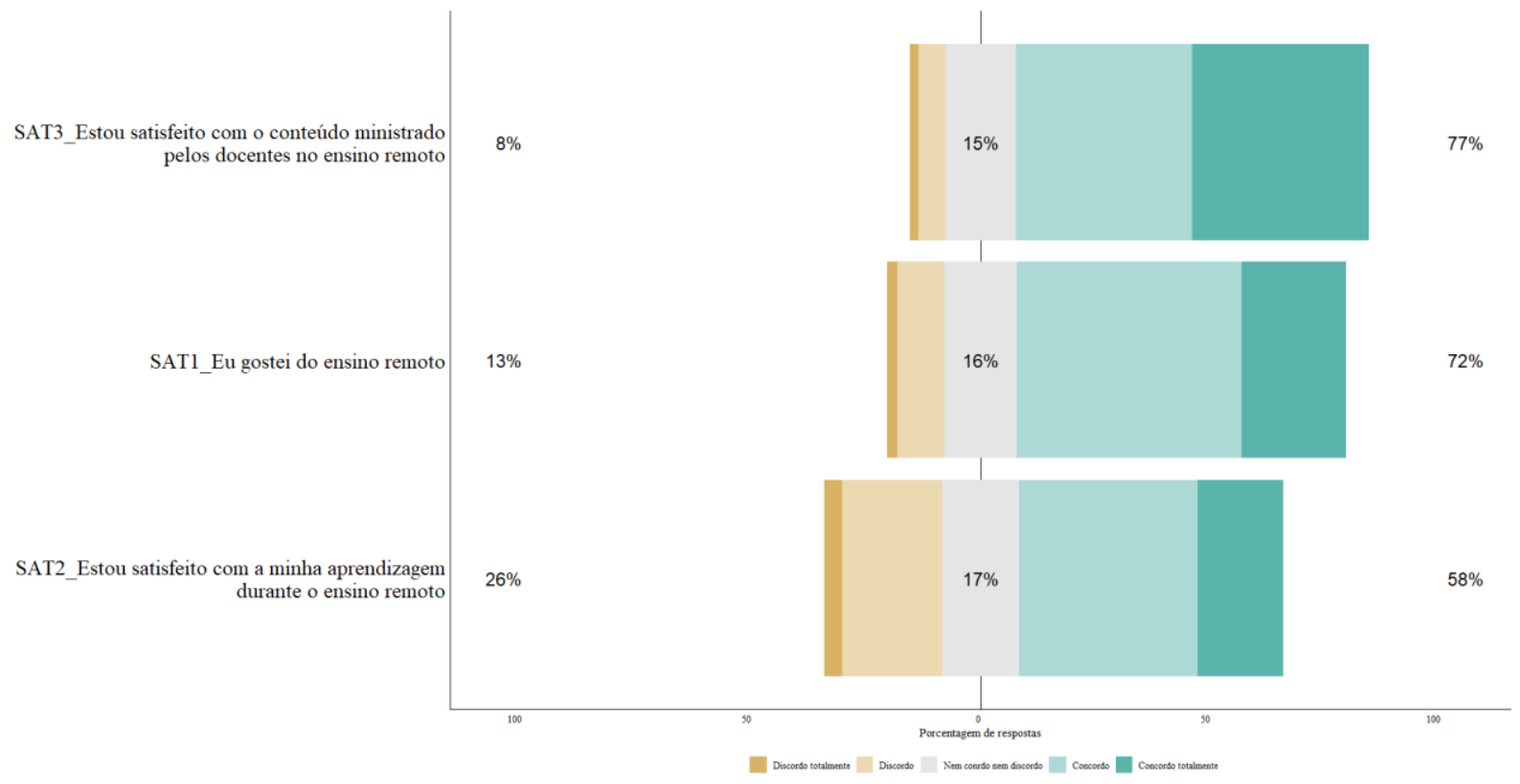

Fonte: Dados da pesquisa, 2021.

4.2 MODELO DE MENSURAÇÃO 
O modelo de mensuração define a forma como os construtos ou variáveis latentes são operacionalizados pelas variáveis observadas ou manifestas. Omodelo utilizado seguiu as recomendações de Wold (1982, p. 41), ou seja, os indicadores das Variáveis Latentes $(\mathrm{VL})$ de $1^{\underline{a}}$ ordem foram reutilizados como indicadores reflexivos da VL de segunda ordem também.

Inicialmente, foram avaliados os resultados dos critérios relacionados à validade convergente das VL de primeira e segunda ordens. Fornell e Larcker (1981) sugeriram utilizar a AVE como critério de validade convergente. Um AVE de pelo menos 0,5 indica que a variável latente é capaz de explicar, em média, mais da metade da variância de seus indicadores. No caso das variáveis de segunda ordem, a confiabilidade composta e a AVE foram calculadas considerando as cargas fatoriais das variáveis de primeira ordem (BIDO; SILVA, 2019). Conforme Hair Jr. et al. (2014), os indicadores com cargas fatoriaismenores que 0,708 devem ser retirados do modelo pois influenciam no valor variância média extraída (AVE), com exceção dos indicadores HAB3 e DOC4 queapresentaram, respectivamente, os valores 0,244 e 0,590 , todos os outros indicadores foram mantidos e, portanto, válidos para compor as VL do modelo.

Para avaliar a confiabilidade do modelo utilizou-se o Alfa de Cronbach e o Rho de Dillon-Goldstein (Tabela 1). Ambos os índices mensuram a unidimensionalidade das variáveis latentes. Para Tenenhaus et al. (2005), uma variável latente apresentará unidimensionalidade quando o Alfa de Cronbach for superior a 0,7 e/ou o Rho de Dillon-Goldstein for igualmente superior a 0,7, conforme Tabela 1.

Tabela 1 - Critérios relacionados à qualidade da consistência interna das VL de $1^{\underline{a}}$ ordem

\begin{tabular}{l|l|l}
\hline Variável latente & Alfa de Cronbach & Rho de Dillon-Goldstein \\
\hline Aprendizagem & 0,749 & 0,751 \\
\hline Docentes & 0,752 & 0,754 \\
\hline Interação & 0,877 & 0,879 \\
\hline Habilidade & 0,743 & 0,743 \\
\hline Satisfação & 0,773 & 0,781 \\
\hline Tecnologia & 0,914 & 0,914 \\
\hline
\end{tabular}

Fonte: Dados da pesquisa, 2021.

Para avaliar a validade discriminante utilizou-se o critério de Fornell e Larcker (1981), que postula que a raiz quadrada da AVE de cada variável deve ser maior que a mais alta correlação da variável com qualquer outra variável latente. 
Tabela 2 - Critério de Fornell-Larcker relacionado à validade discriminante

\begin{tabular}{|c|c|c|c|c|c|c|c|c|}
\hline VL de 1 ${ }^{\text {a }}$ ordem & 1 & 2 & 3 & 4 & 5 & 6 & 7 & 8 \\
\hline 1 - Aprendizagem & $\begin{array}{c}0,81 \\
6\end{array}$ & & & & & & & \\
\hline 2 - Docentes & $\begin{array}{c}0,77 \\
3\end{array}$ & $\begin{array}{c}0,81 \\
8\end{array}$ & & & & & & \\
\hline 3 - Interação & $\begin{array}{c}0,72 \\
1\end{array}$ & $\begin{array}{c}0,69 \\
3\end{array}$ & $\begin{array}{c}0,89 \\
6\end{array}$ & & & & & \\
\hline 4 - Habilidade & $\begin{array}{c}0,76 \\
6\end{array}$ & $\begin{array}{c}0,73 \\
7\end{array}$ & $\begin{array}{c}0,65 \\
6\end{array}$ & $\begin{array}{c}0,89 \\
2\end{array}$ & & & & \\
\hline 5 - Satisfação & $\begin{array}{c}0,72 \\
6\end{array}$ & $\begin{array}{c}0,68 \\
6\end{array}$ & $\begin{array}{c}0,75 \\
8\end{array}$ & $\begin{array}{c}0,68 \\
3\end{array}$ & $\begin{array}{c}0,83 \\
0\end{array}$ & & & \\
\hline 6 - Tecnologia & $\begin{array}{c}0,68 \\
1 \\
\end{array}$ & $\begin{array}{c}0,65 \\
0\end{array}$ & $\begin{array}{c}0,79 \\
4\end{array}$ & $\begin{array}{c}0,64 \\
3\end{array}$ & $\begin{array}{c}0,80 \\
2\end{array}$ & $\begin{array}{c}0,89 \\
1\end{array}$ & & \\
\hline \multicolumn{9}{|l|}{ VL de $2^{\text {a }}$ ordem } \\
\hline 7 Características Individuais & & & & & & & $\begin{array}{c}0,81 \\
8\end{array}$ & \\
\hline 8 Qualidade & & & & & & & 0,480 & $\begin{array}{c}0,75 \\
8\end{array}$ \\
\hline Confiabilidade composta & $\begin{array}{l}0,85 \\
7\end{array}$ & $\begin{array}{c}0,85 \\
8\end{array}$ & $\begin{array}{c}0,92 \\
5\end{array}$ & $\begin{array}{c}0,88 \\
6\end{array}$ & $\begin{array}{c}0,86 \\
9\end{array}$ & $\begin{array}{c}0,93 \\
9\end{array}$ & $\begin{array}{c}0,92 \\
6\end{array}$ & $\begin{array}{c}0,93 \\
7\end{array}$ \\
\hline AVE & $\begin{array}{c}0,66 \\
6\end{array}$ & $\begin{array}{c}0,66 \\
9\end{array}$ & $\begin{array}{c}0,80 \\
4\end{array}$ & $\begin{array}{c}0,79 \\
6\end{array}$ & $\begin{array}{c}0,68 \\
8\end{array}$ & $\begin{array}{c}0,79 \\
5\end{array}$ & $\begin{array}{c}0,86 \\
3\end{array}$ & $\begin{array}{c}0,83 \\
2\end{array}$ \\
\hline
\end{tabular}

Nota: Valores na diagonal são a raiz quadrada da AVE e os demais valores são as correlações entre as variáveis

Antes de iniciar a análise do modelo estrutural, avaliou-se a colinearidade (VIF), tendo em vista que os coeficientes do caminho podem ser tendenciosos sea estimativa envolver níveis significativos de colinearidade entre os construtos preditores. Todas as variáveis apresentaram valores VIF menor que o limite 5, portanto, entende-se que o critério foi atendido.

\subsection{MODELO ESTRUTURAL}

Por meio do procedimento de bootstrapping, calculou-se os coeficientes de caminho que representam as relações hipotéticas entre os construtos e têm similaridades com outros modelos de regressão tradicionais, a diferença é que eles apresentam, antes de qualquer coisa, as relações estruturais (AMORIM, 2012). $\mathrm{Na}$ tabela 3 é possível perceber que a maior ligação é entre a Qualidade percebida e satisfação $(0,651)$.

Tabela 3 - Resultado do Teste de significância dos Coeficientes de caminho

\begin{tabular}{lccccc}
\hline $\begin{array}{l}\text { Relação entre os } \\
\text { constructos }\end{array}$ & $\begin{array}{c}\text { Coeficiente } \\
\text { original }\end{array}$ & $\begin{array}{c}\text { Média da } \\
\text { amostra }\end{array}$ & $\begin{array}{c}\text { Desvio } \\
\text { padrão }\end{array}$ & Teste t & p value \\
\hline $\begin{array}{l}\text { Características Individuais -> } \\
\begin{array}{l}\text { Satisfação } \\
\text { Qualidade percebida - }\end{array}\end{array} 0,212$ & 0,213 & 0,076 & 2,788 & 0,005 \\
>Satisfação & 0,651 & 0,650 & 0,073 & 8,961 & 0,000 \\
\hline
\end{tabular}

Fonte: Dados da pesquisa, 2021.

Ainda de acordo com a Tabela 3 , verifica-se que os coeficientes calculadossão 
significativos ao nível de $5 \%$ ( $p$ value $<0,05)$, assim, as duas hipóteses informadas anteriormente foram validadas. Em seguida, avaliou-se outros indicadores de qualidade como relevância preditiva $\left(\mathrm{Q}^{2}\right)$ e tamanho de efeito $\left(\mathrm{f}^{2}\right)$. Todos os valores de $Q^{2}$ encontrados foram maiores que zero, o que permite afirmar que o modelo ajustado tem alto grau de relevância preditiva. Já os valores de $\mathrm{f}^{2}$ avaliam a importância relativa de cada construto no modelo, sendo assim, ovalor encontrado entre características individuais e satisfação $(0,035)$ foi considerado pequeno e entre qualidade e satisfação (0,332) é considerado médio(HAIR JR et al, 2014).

A partir das análises realizadas e dos resultados obtidos, apresenta-se na Figura 04, o modelo de mensuração e estrutural. Avaliando o valor do $\mathrm{R}^{2}$, que corresponde a uma medida da acurácia preditiva e representa os efeitos combinados das variáveis latentes exógenas na variável latente endógena (HAIRJR et al., 2014), obteve-se o valor de 0,711 sugerindo um efeito elevado. As relações entre a VL de segunda ordem e suas dimensões (VL de primeira ordem)devem ser interpretadas e usadas como cargas fatoriais e, portanto, não são hipóteses (BIDO; SILVA, 2019). A partir dos resultados relatados, observa-se quetodas as hipóteses foram aceitas apresentando valores $\mathrm{t}<1,96$ e p>0,05 para um nível de significância de 5\%.

Figura 4 - Modelo de mensuração e estrutural

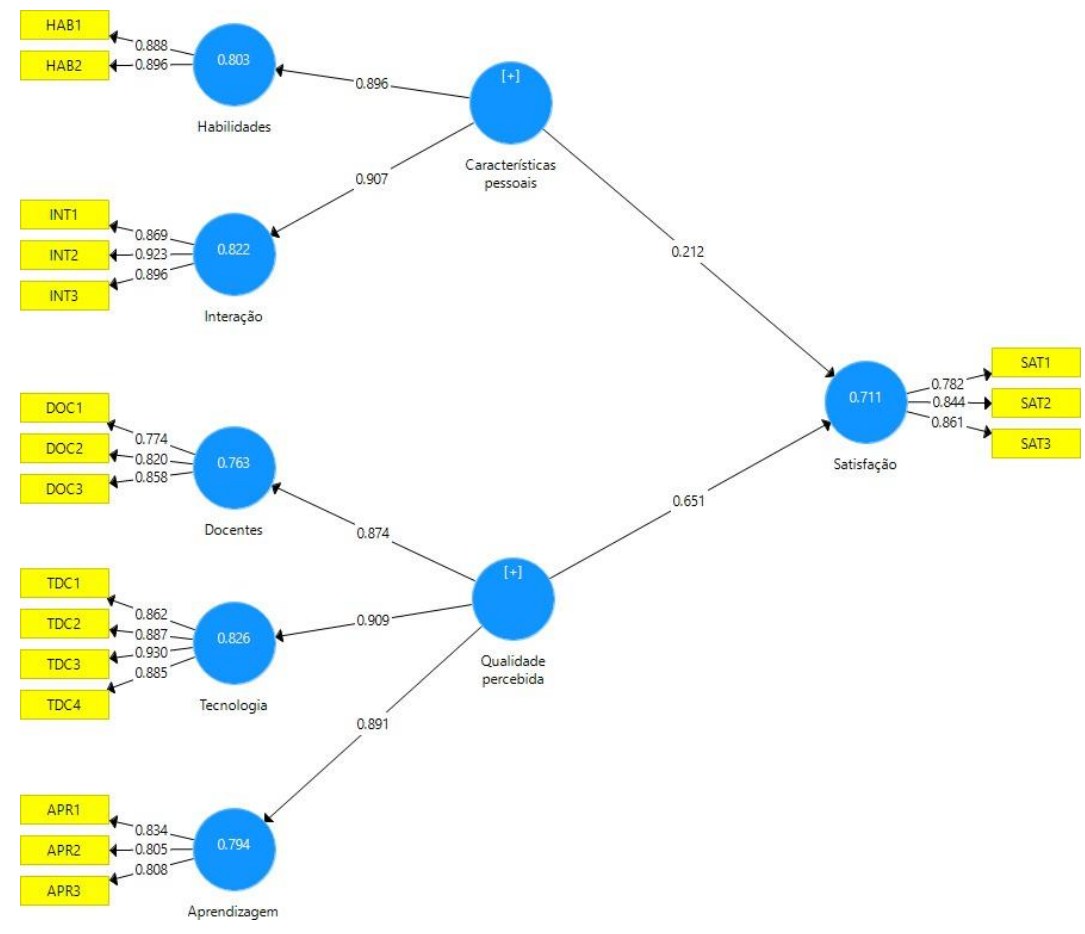

Fonte: Dados da pesquisa, 2021. 


\subsection{ANÁLISE E DISCUSSÃO DOS RESULTADOS}

Detalhando a análise para cada hipótese, na Figura 1 é possível visualizarque conforme $\mathrm{H}_{1}$ : Há uma relação positiva entre a percepção de qualidade e a satisfação dos estudantes com o ER, e logo, entende-se que a percepção da qualidade, mensurada pelas práticas docentes, TDIC e aprendizagem adquirida, é o principal fator que influencia na satisfação dos estudantes com o $E R$, tendo em vista o valor do coeficiente de caminho encontrado $(0,651)$. Esse resultado corrobora com os estudos de Abbasi et al. (2020) a respeito da importância do docente, diante desta situação inesperada de pandemia, em que o ensino foi transferido para o sistema remoto a fim de garantir a continuidade das atividades acadêmicas. As TDIC também influenciaram na percepção da qualidade do ER (ELUMALAl et al.; 2020; ALDHAHI, 2021).

Evidenciou-se também a aprovação de $\mathrm{H}_{2}$, e, portanto, há uma relação positiva entre as características individuais dos estudantes e a sua satisfação como ER. As habilidades e competências dos estudantes em utilizar recursos tecnológicos e redes sociais e as características de interação e colaboração influenciam a satisfação com o ER. Trata-se de um ambiente onde a interação e a comunicação entre o docente e o estudante, e inclusive em meio aos próprios estudantes, melhora a qualidade do aprendizado (GOH et al., 2017; YEKEFALLAH et al., 2021).

\section{CONCLUSÃO}

Através da análise realizada no modelo estrutural e no instrumento utilizadoé possível afirmar que todos os objetivos específicos foram alcançados, tendo emvista o entendimento do perfil do estudante on-line no Brasil ora apresentado, bemcomo as variáveis que influenciam a satisfação do estudante universitário com o ER, diante de um cenário de pandemia. Após o desenvolvimento dos testes de confiabilidade e validade, que foram medidos pelo programa SmartPls, foi possível responder ao problema de pesquisa: Em função da necessidade de distanciamento social, e a implantação de um regime excepcional de oferta de disciplinas, quais as variáveis foram determinantes para a satisfação dos estudantes com o ER?

Para responder à questão de pesquisa proposta este estudo identificou o 
grau de concordância dos estudantes com aspectos que avaliam o ER, destacando-se o papel dos docentes em assegurar que o processo de ensino e a aprendizagem ocorresse, por meio do uso das TDIC, permitindo assim que os cursos de graduação fossem continuados, mesmo em um cenário adverso e imprevisível. Evidenciou-se ainda que, os estudantes pesquisados são jovens, com idade média de 23 anos e usuários das redes sociais, acostumados com o uso de ferramentas e recursos digitais e gostam de colaborar e trabalhar em equipe.

As hipóteses $\mathrm{H}_{1}$ : há uma relação positiva entre a percepção de qualidade e a satisfação dos estudantes e $\mathrm{H}_{2}$ : há uma relação positiva entre as características individuais dos estudantes e com o ER, foram confirmadas. Sendoa percepção da qualidade $(65,1 \%)$ mais importante que as características individuais $(21,2 \%)$, ou seja, fatores relacionados às práticas docentes, tecnologia e percepção de aprendizagem melhoram a satisfação com o ER.

Tendo como referência o $\mathrm{R}^{2}$, que corresponde a uma medida de previsão e representa os efeitos combinados das variáveis latentes exógenas na variável latente endógena obteve-se como o valor de 0,71 sugerindo um efeito substancial. Assim, infere-se que o objetivo deste estudo foi alcançado, e pode ser utilizado como base para estudos futuros. 


\section{REFERÊNCIAS}

Abbasi, s. ; ayoob, t.; malik, a. ; memon, s. I. Perceptions of students regarding elearning during covid-19 at a private medical college. Pakistan journal ofmedical $\begin{array}{lllll}\text { sciences, } & 36 & \text { (covid19-s4), } & \text { 57-61, } & \end{array}$ https://doi.org/10.12669/pjms.36.covid19-s4.2766.

Adams becker, s.; cummins, m. ; davis, a.; freeman, a.; hall giesinger, c.; ananthanarayanan, v. Nmc horizon report: 2017, higher education edition. New media consortium. Disponível em: $<$ https://www.sconul.ac.uk/sites/default/files/documents/2017-nmc-horizonreport-he-en.pdf>. Acesso em: 20 set. 2021.

Aldhahi, m.; alqahtani, a.; baattaiah, b.; almohammad, h. Exploring the relationship between students' learning satisfaction and self-efficacy during the emergency transition to remote learning amid the coronavirus pandemic: a cross-sectional study. Education and information technologies, 2021 doi: 10.1007/s10639-02110644-7.

Amorim, I. D. A. F. Et al. Modelagem com equações estruturais: princípios básicose aplicações. Salvador: universidade federal da bahia. Instituto de matemática, 2012. $49 \mathrm{p}$.

Arruda e. P. Educação remota emergencial: elementos para políticas públicas na educação brasileira em tempos de covid-19. Em rede, 7 (1), 257-75, 2020.

Ashwin, p. ; mcvitty, d. The meanings of student engagement: implications for policies and practices. In: curaj, a.; matei, I.;pricopie, r.; salmi, j.; scott, p. (eds.). The european higher education area (p. 343-359). Springer, 2015. Https://doi.org/10.1007/978-3-319-20877-0_23.

Atchley, w.; wingenbach, g.; akers, c. Comparison of course completion and student perfor-mance through online and traditional courses. International review of research in open and dis-tributed learning,14(4), 104-116, 2013

Bido, d. S.; silva, d. Smartpls 3: especificação, estimação, avaliação e relato. Revista de administração: ensino e pesquisa (raep), v. 20, n. 2, p.1-31, 2019. Doi: 10.13058/raep.2019.v20n2.1545.

Bolliger, d. U. Key factors for determining student satisfaction in online courses. International journal on e-learning, 3(1), 61-67. Association for the advancement of computing in education (aace). Retrieved from https:// www. Learn techl ib. Org/ prima ry/p/ 2226/., 2004

Cole, m.t. ; shelley, d.j.; swartz, I.b. Online instruction, e-learning, and student 
satisfaction: a three-year study. The international review of research in open and distributed learning, 15(6). Irrodl. V15i6. 1748, 2014. Https:// doi. Org/ 10. 19173/

Debattista, m. A comprehensive rubric for instructional design in e-learning. International journal of information and learning technology, 35(2), 93-104, 2018. Https://doi.org/10.1108/ijilt-09-2017-0092.efron, b.; tibshirani, r. J. An introduction to the bootstrap, chapman hall: new york, 1993.

Elumalai, k. V.; sankar, j. P.; john, j. A.; menon, n.; alqahtani, m. S. M.; abumelha, $\mathrm{m}$. A. Factors affecting the quality of e-learning during the covid-19 pandemic from the perspective of higher education students. Journal of information technology education: research, v. 19, 731-753, 2020. Doi: https://doi.org/10.28945/4628.

Esfijani, a. Measuring quality in online education: a meta-synthesis. The american journal of distance education, , v 32 (1), p. 57-73, 2018.

Fornell, c. ; david f. L.r. Evaluating structural equation models with unobservable variables and measurement error. Journal of marketing research, vol. 18, no. 1, american marketing association, 1981, pp. 39-50, https://doi.org/10.2307/3151312.

Garcia-alberti, m.; suarez, f.; chiyon, i.; feijoo, j. C. M. Challenges and experiencesof online evaluation in courses of civil engineering during the lockdown learning due to the covid-19 pandemic. Education sciences., 11, 59, 2021. Doi: https://doi.org/10.3390/educsci11020059.

Gelles, I. ; lord, s.; hoople, g.; chen, d.; mejia, j. Compassionate flexibility and selfdiscipline: student adaptation to emergency remote teaching in an integrated engineering energy course during covid-19. Education sciences, 10, 304, 2020. 10.3390/educsci10110304.

Goh, c. F.; leong, c. M.; kasmin, k.; hii, p. K.; tan, o. K. Students' experiences, learning outcomes and satisfaction in e-learning. Journal of e-learning and knowledge society, 13(2), 117-128, 2017. Https://doi.org/10.20368/1971$8829 / 1298$.

Hair jr, j. F.; gabriel, m. L. D. S.; patel, v. K. Modelagem de equações estruturais baseada em covariância (cb-sem) com o amos: orientações sobre a sua aplicação como uma ferramenta de pesquisa de marketing. Revista brasileira de marketing,v. 13, n. 2, p. 44-55, 2014. Doi: 10.5585/remark.v13i2.2718.

Hair,, j. F.; hult, g. T. M.; ringle, c. M.; sarstedt, m. A primer on partial least squares structural equation modeling (pls-sem). Sage: thousand oaks, 2. Ed, 2017. 
Hodges, c.; more, s.; lockee, b.; trust, t.; bond, a. The difference between emergency remote teaching and online learning. Educause review, 27 mar. 2020. Disponível em: https://er.educause.edu/articles/2020/3/the-difference-betweenemergency-remoteteaching-and-online-learning, 2020. Acesso em: 05 mar, 2021

Jung, i. The dimensions of e-learning quality: from the learner's perspective. Educational technology research and development, vol. 59, n. 4, p. 445-464, aug. 2011.

Lima n. C.; souza g. S.; queiroz, f. C. B. P. Queiroz, j. V.; silva C. L. Covid-19 no brasil: explorando dinâmicas econômicas e sociais. Espacio abierto: cuaderno venezolano de sociología, issn 1315-0006, vol. 29, no․ 4, ,págs. 43-69, 2020

Maki, r.h., maki, w.s., patterson, m. Et al. Evaluation of a web-based introductory psychology course: i. Learning and satisfaction in on-line versus lecture courses. Behavior research methods, instruments, \& computers 32, 230-239. 2000. Https://doi.org/10.3758/bf03207788

Nicola, m.; alsafi, z.; sohrabi, c.; kerwan, a.; al-jabir, a.; iosifidis, c.; agha, m.; agha, $r$. The socio-economic implications of the coronavirus and covid-19 pandemic: a review. International journal of surgery, journal pre-proof, 12 de abr. 2020. Disponível em: <https://doi.org/10.1016/j.ijsu.2020.04.018>. Acesso em: 28 set. 2021.

Puriwat, w.; tripopsakul, s. The impact of e-learning quality on student satisfaction and continuance usage intentions during covid-19. International journal of information and education technology, 11(8): 368-374, 2021.

Rahiem, m. D. H. Technological barriers and challenges in the use of ict during the covid-19 emergency remote learning. Universal journal of educational research, v.8, n. 11b, p. 6124-6133, 2020. Doi: 10.13189/ujer.2020.082248

Saxena, c.; baber, h.; kumar, p. Examining the moderating effect of perceived benefits of maintaining social distance on e-learning quality during covid-19 pandemic, journal of educational technology systems, 49(4), p. 532-554, 2021. Doi: $10.1177 / 0047239520977798$.

Seiver, j. G.; troja, a. Satisfaction and success in online learning as a function of the needs for affiliation, autonomy, and mastery. Distance education, 35(1), 90105, 2014. Https:// doi. Org/10. 1080/ 01587 919. 2014. 891427

Souza, s. R. S.; choma neto, j.; paschoal, I. N.; hernandes, e. Ensino remoto 
emergencial de engenharia de software com pbl: um relato de experiência. In: workshop sobre educação em computação (wei), 29, 2021, evento online. Anais [...]. Porto alegre: sociedade brasileira de computação, 2021, p. 31-40. Issn 2595-6175. Doi: https://doi.org/10.5753/wei.2021.15894.

Sun,p. C.; tsai, r. J.; finger, g.; chen, y. Y.; yeh, d. What drives a successful elearning? An empirical investigation of the critical factors influencing learner satisfaction. Computers \& education, v. 50, 4 ed., p. 1183-1202, 2008. Doi : https://doi.org/10.1016/j.compedu.2006.11.007.

Tenenhaus, m. ; vinzi, v.; chatelin, y. ; et al. Pls path modeling. Computational $\begin{array}{lllll}\text { statistics data } & \text { analysis, 48, 159-205, }\end{array}$ https://doi.org/10.1016/j.csda.2004.03.005

Wold, h.o.a. Soft modeling: the basic design and some extensions. In: joreskog, k.g. And wold, h.o.a., eds., systems under indirect observations: part ii, northholland, amsterdam, 1-54, 1982

Yekefallah, I.; namdar, p.; panahi, r.; dehghankar, I. Factors related to students' satisfaction with holding e-learning during the covid-19 pandemic based on the dimensions of e-learning. Heliyon, v. 7, 7 ed, e07628, 2021. Doi : 10.1016/j.heliyon.2021.e07628.

Yu, j.; huang, c.; han, z.; he, t.; li, m. Investigating the influence of interaction on learning persistence in online settings: moderation or mediation of academic emotions? International journal of environmental research and public health, 17(1), 1-21, 2020. Https://doi.org/10.3390/ijerph17072320. 